\section{MS34-P4 Ferrocene derivatives as potential mechanophores for stimuli-responsive materials}

Michela Di Giannantonio ${ }^{1}$, Katharina M. Fromm ${ }^{1}$

1. Departement of Chemistry, University of Fribourg, Chemin du Musée 9, CH-1700 Fribourg, Switzerland

email: michela.digiannantonio@unifr.ch

Since long time ferrocene and its derivatives have attracted the attention of the scientific and technical community because of its fascinating chemistry. Due to its easy functionalization and unusual and attractive properties, ferrocene derivatives have found different applications in material science, such as sensors, catalysts, polymers, electro active materials and medicinal chemistry ${ }^{1,2}$. The aim is to synthesize linear polyurethane chains or linear poly(methyl methacrylate) with a number of covalently linked ferrocene units in the backbone. Upon exerting a mechanical stress (e.g. sonication in solution), we expect the polymer to break along the weakest bonds, i.e. the ferrocene moieties. In order to verify this hypothesis, we analyze the physico-chemical properties and changes of the polymers following sonication in solution or using techniques such as the atomic force microscope (AFM), in order to understand their potential as stimuli-responsive materials.

In collaboration with Prof. C. Weder (Adolphe Merkle Institute, Fribourg) the first mechanical tests in solution were performed with the linear polyurethane ( $\mathrm{Fc} 3 \%$ ) exploiting the ultrasound-induced degradation ${ }^{3}$ to stretch the polymer and to selectively break the weak bonds. With the first results obtained by a kinetic study (Fig. 1) we can conclude that the ferrocene moiety could be a potential mechanophore in a linear polymer. The synthesized ferrocene-based polymers will be further studied exploiting the $\mathrm{AFM}^{4}$ and other techniques to confirm these preliminary findings.

References: ${ }^{1}$ S. Zhai, J. Shang, D. Yang, S.Wang, J. Hu, G. Lu, X. Huang, J. Polym. Sci. Part Polym.Chem. 2012, 50, 811-820.

2 C. Herfurth, D. Voll, J. Buller, J. Weiss, C. Barner-Kowollik, A. Laschewsky, J. Polym. Sci. Part Polym.Chem. 2012, 50, 108-118.

${ }^{3}$ M. M. Caruso, D. A. Davis, Q. Shen, S. A. Odom, N. R. Sottos, S. R. White, J. S. Moore, Che,. Rev. 2009, 109, 5755-5798.

4 N. Hosono, A. M. Kushner, J. Chung, A. R. A. Palmans, Z. Guan, E. W. Meijer, JACS, 2015, 137, 6880-6888.
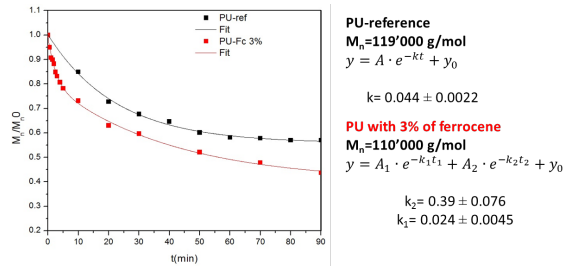

Figure 1. Ultrasound-induced degradation of a Fc-containing PU.

\section{MS34-P5 Multitopic precursors for oxide materials' synthesis.}

Alba Finelli ${ }^{1}$, Aurélien Crochet ${ }^{2}$, Katharina M. Fromm ${ }^{3}$

1. Department of Chemistry, University of Fribourg, Chemin du Musée 9, 1700 Fribourg.

2. FriMat, Department of Chemistry, University of Fribourg, Chemin du Musée 9, 1700 Fribourg.

3. Department of Chemistry, University of Fribourg, Chemin du Musée 9, 1700 Fribourg.

email: alba.finelli@unifr.ch

The research interest in mixed metal oxides is increasing in material science, as they have multiple applications, such as in batteries, ceramics, pigments, high-Tc superconductors or transparent conductors. However, the two main challenges for the synthesis of such compounds are the lack of control on the ratio of the different metal components and the extreme conditions (up to $900{ }^{\circ} \mathrm{C}$ ) that many of these oxides require during their traditional solid state synthesis. To overcome these issues, we propose a strategy for the synthesis of mixed metal complexes, which is based on precursors of coordination compounds, using the "multitopic ligand approach". The aim is to design specific ligands with selective coordination sites to bind different metal ions. Due to the metal ion preorganization in the precursor thus formed, the stoichiometry of the final oxide material can be controlled and the extreme synthesis conditions diminished (pressure or temperature). These new mixed metal complexes will be finally combusted to oxide materials with possible new features and ideally at the nanoscale, allowing to access new and better properties in their applications.

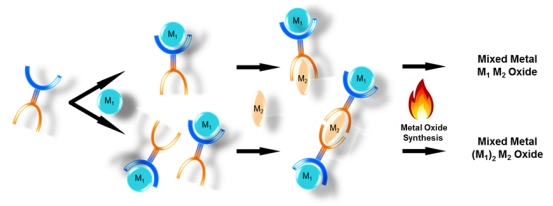

Figure 1. Multitopic ligand approach

Keywords: Inorganique chemistry, complexes, alkali, alkaline earth

Keywords: polyurethanes, ferrocene, sonication 